\title{
Detection of fatty product falsifications using a portable near infrared spectrometer
}

\author{
A.V. Kalinin*, and V.N. Krasheninnikov \\ Institute for Spectroscopy RAS, 108840 Troitsk, Moscow, Russia
}

\begin{abstract}
Spreading sales of counterfeited fatty-oil foods leads to a development of portable and operational analyzer of typical fatty acids (FA) which may be a near infrared (NIR) spectrometer. In this work the calibration models for prediction of named FA were built with the spectra of FT-NIR spectrometer for different absorption bands of the FA. The best parameters were obtained for the wavelength sub-band 1.0-1.8 $\mu$, which includes the 2 nd and $3 \mathrm{rd}$ overtones of $\mathrm{C}-\mathrm{H}$ stretching vibrations (near 1.7 and $1.2 \mu)$ and the combination band $(1.42 \mu)$. Applicability of the portable spectrometer based on linear NIR array photosensor for the quality analysis of spread, butter and fish oil by the typical FA has been tested.
\end{abstract}

Determination of individual fatty acids (FA), and the groups of them (cis-, trans-, saturated, unsaturated, and other) in food products and supplements have been made in recent studies [1-2] using FT spectrometer in the near infrared (NIR) spectral region for a variety of overtones and combinations of fundamental vibrations of $\mathrm{C}-\mathrm{H}, \mathrm{C}=\mathrm{C}, \mathrm{C}=\mathrm{O}$ with the use of multivariate calibration (regression) on projections to latent structures (PLS). Efficiency of FA prediction using different absorption bands of overtones and combinations in these works has not been studied, which prevents the selection of optimal technical conditions for creation of portable NIR spectrometer, eg with the linear InGaAs array photosensor. The aim of this work was to study the efficiency of calibration models built with different FA absorption bands, and the applicability of NIR spectrometer with linear G9213S photosensor, Hamamatsu, Japan, as a prototype of a portable quality analyzer of spread, butter and fish oil on content of FA: butyric C4:0, myristic C14:0, palmitic C16:0, oleic $\mathrm{C} 18: 1$, and the amount of polyene $\Omega 3 \mathrm{C} 20: 5+\mathrm{C} 22: 6$.

Spectroscopic analysis involves time-consuming multi-component calibration over a large number of calibration samples. In previous work [3], we have developed costeffective technique that provides a good calibration model with a special choice of variations of three components in the set of only 35 samples. A similar design was done in the first set of 50 mixtures of milk fat, palm and sunflower oils to determine the content of FA C4:0, C14:0 and C16:0, and in the second set of 112 samples to determine the content of C16: 0, C18: 1 and C20: 5 C22 + 6. Calibration (35, 38 and 39 units) and testing (35 and 25 units, respectively) sets of samples were standardized using the thin-film gas chromatograph Kristallyuks4000M Ltd. Interchrom, RF, in accordance with ISO 5508: 1990 (E) .This samples can serve also as standards of milk fat, palm, olive and fish oil fractions, from which weighed portions they were manufactured. Pretreatment of calibration samples and tested products was performed taking into account the testing

\footnotetext{
* Corresponding author: kalinin@isan.troitsk.ru
} 
results of several methods for fat extraction, obtained in [2]. Mixtures of oils and fats in the test tube was heated to $60^{\circ} \mathrm{C}$ and held for sedimentation, then the lower fraction was drained through the hole at the bottom, and the top foam, if any, was filtered in a special incubator as described in [3]. FA ranges of variations were in the first set of samples for C4:0 (0.5-4), for C14:0 (6-14), for C16:0 (14-33), in the second set for C16:0 (14- 35), for C18:1 (24-47), and for C22 5+C24:6 (12-25)\% of total fat.

Absorption spectra of second set samples were acquired first using FTNIR spectrometer MPA Bruker, Germany in the wavelength range 0.9-2.2 $\mu$, and, secondly, via a fiber-optic InGaAs spectrometer within the range 0.9-1.65 $\mu$ (see Fig. 1). Built on two sets of samples PLS models were tested with the samples of not less than 25 standards that were not used in the calibration model. Models with the spectra of the FTNIR spectrometer for four spectral sub-regions indicate the similar errors, but the model for IGA spectrometer in the range 1.1-1.64 $\mu$ has the lowest total error, in comparison with the models for full range of FTNIR spectrometer. The best tested models (standard error of $0.49,1.79$ and 0.51 for the $\mathrm{C} 16: 0, \mathrm{C} 18: 1$ and $\Omega 3$, respectively) then were used for analyses of certain foods and supplements, the results are presented in Table 1.

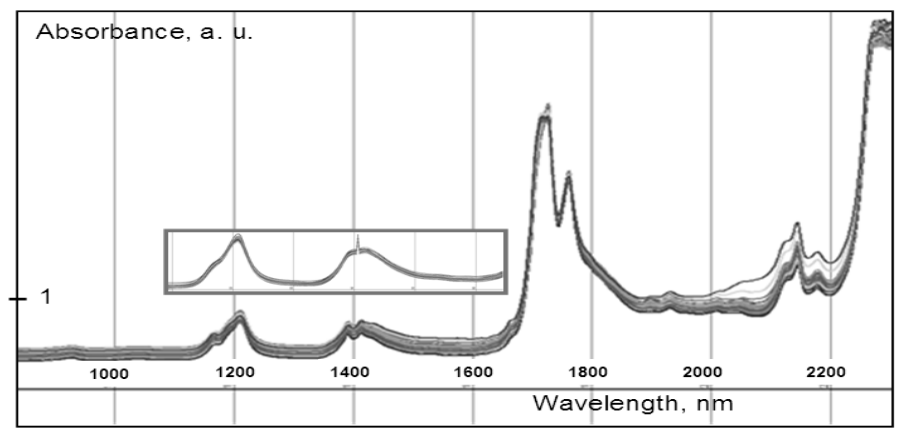

Fig. 1. Absorbance spectra of 51 fat-oil mixtures in the test tube $\varnothing 6 \mathrm{~mm}$ obtained by FTNIR spectrometer MPA Bruker. Spectra of the same samples accumulated with IGA spectrometer (insert).

Table 1. Prediction of the content of typical FA in spreads and butter and. $\Omega 3$ FA in dietary supplements and fish oils

\begin{tabular}{|l|l|l|l|l|l|l|}
\hline \multirow{2}{*}{ Product Value } & \multicolumn{2}{|l|}{ FA fraction } & Conclusion \\
\cline { 2 - 7 } & $\mathrm{C} 4: 0$ & $\mathrm{C} 14: 0$ & $\mathrm{C} 16: 0$ & $\mathrm{C} 18: 1$ & $\Omega 3$ & \\
\hline $\begin{array}{l}\text { Supplement Fish Oil } \\
\text { " } \Omega 3 \text { " }\end{array}$ & $<.5$ & & 2.4 & 39.9 & 24.5 & Normal \\
\hline Oil of Raw Capelin & $<.5$ & & 15.9 & 41.2 & 13.1 & \multicolumn{1}{|l|}{} \\
\hline Oil of Baked Capelin & $<.5$ & & 14.2 & 410. & 11.1 & $\begin{array}{l}\text { Less than 15\% } \\
\text { raw }\end{array}$ \\
\hline Spread «Kremliovskoye» & 1.1 & 6.1 & 29.2 & & & $\begin{array}{l}\text { Shortage of milk } \\
\text { fat }\end{array}$ \\
\hline Spread «Extra»,72\% & $<.5$ & 12.2 & 30.3 & & & $\begin{array}{l}\text { Shortage of milk } \\
\text { fat }\end{array}$ \\
\hline Butter "Valio", 72\% & 2.2 & 8.7 & 32.4 & & & Normal \\
\hline Butter «Is Vasilcova» & $<.5$ & & & & & Falsed \\
\hline Milkfat substitute "Efko" & 1.5 & 2.6 & 44. & & & Milk fat less 30\% \\
\hline
\end{tabular}

Models derived from the spectra accumulated by the IGA spectrometer have the same accuracy and reliability of determining the content of C16:0 and C18:1, compared with the models on the Fourier spectra to estimate the proportion of milk fat in spreads published in [2]. Determination of characteristic FA helped to draw conclusions on the percentage of milk fat in the milk-containing products and the content of FA $\Omega 3$ in fish oil. 
Conclusions: a) the content of FA C4: 0, C14: 0 and C16: 0 using InGaAs spectrometer were well defined in mixtures where milk fat percentage was of $15 \%$ or higher; b) FA $\Omega 3$ were well-defined by all models; c) the best parameters for determining FA C16:0, C18:1 using FTNIR spectrometer in the spectral range 0.9-2.2 $\mu$ had the model for the sub-band 1.1-1.8 $\mu$, which includes the 2 nd and 3 rd overtone of $\mathrm{C}-\mathrm{H}$ stretching vibrations (near 1.7 and $1.2 \mu)$ and combination at $1.42 \mu$. According to the concepts of atherosclerosis evolution [4] the contents of C16: 0 and C18: $1 \mathrm{FA}$ in food and the serum of patients has special diagnostic significance. Creating a InGaAs spectrometer for the 0.9-1.8 $\mu$ spectral region, will allow, perhaps, to improve the methodology of FA determination and to realize portable FA analyzer for operational monitoring of fish, meat and dairy products and dishes.

\section{References}

1. http://lipidlibrary.aocs.org/topics/IR-trans/index.htm

2. G. Cabassi et al., J. Near Infrared Spectrosc. 21, 395 (2013)

3. A. Kalinin et al. J. Near Infrared Spectrosc. 21, 409 (2013)

4. V.N. Titov, Pathogenesis 11, 18 (2013) 